\title{
Nuevos postulados contables en la normatividad mundial
}

Raúl A. Arrarte Mera ${ }^{1}$

Recibido: 30 de abril de 2015

Aprobado: 15 de mayo de 2015

Arrarte, R. (2015). Nuevos postulados contables en la normatividad mundial. Activos, 24, 153-173.

\section{Clasificación JEL}

M-49

\section{Resumen}

En este trabajo examinamos los nuevos postulados básicos a la luz de los principios de la contabilidad generalmente aceptados como tradicionales. El trabajo aborda una perspectiva analítico-explicativa-documental y de síntesis bibliográfica de libros, revistas contables nacionales e internacionales, compendios y páginas web. La conclusión principal demuestra que se trata de normas dirigidas al inversor, que priorizan lo financiero a lo económico, provenientes del consenso alcanzado por diferentes corrientes teóricas desarrolladas en las últimas décadas, validadas por la práctica de los negocios y cuya finalidad es reflejar la esencia económica de las operaciones y presentar una imagen fiel de la situación financiera de una empresa. Las normas internacionales de información financiera se emiten

1 Doctor en Ciencias Contables y Empresariales. Contador público colegiado por la Universidad Nacional Mayor de San Marcos. Maestro en Administración de la Universidad San Martín de Porres de Lima, Perú. Catedrático principal en la Facultad de Ciencias Contables. Correo electrónico: rarrartem@unmsm.edu.pe 
por la International Accounting Standards Board (IASB) ${ }^{2}$ (anteriormente International Accounting Standards Commite) $)^{3}$.

\title{
Palabras claves
}

Normas, postulados contables, principios contables.

\section{Arrarte, R. (2015). New accounting standards in the world nor- mative. Activos, 24, 153-173.}

\begin{abstract}
This research has the general objective to review the implementation of the new nine basic generally accepted accounting principles. The inquiry is analytical-explanatory documentary, and books, international accounting research journals, abstracts and Web pages bibliographic synthesis. The main finding shows that high quality standards are geared to the investor, give priority to financial economics from the consensus reached by different theoretical approaches developed in recent decades, validated by business practice and intended to reflect the economic substance of operations, and to present a true and fair view of the financial position of the company. IFRS are issued by the International Accounting Standards Board (IASB), (former International Accounting Standards Committee).
\end{abstract}

\section{Keywords}

Standards, accounting postulates, accounting principles.

2 Junta de Estándares Internacionales de Contabilidad

3 Comité de Estándares Internacionales de Contabilidad 


\section{Arrarte, R. (2015). Nouveaux concepts comptables dans la régle- mentation mondiale. Activos, 24, 153-173.}

\section{Résumé}

Dans ce travail nous examinons les nouveaux concepts basiques à la lumière des principes de la comptabilité généralement acceptés comme traditionnels. Le travail aborde une perspective analytique-explicative-documentaire et de synthèse bibliographique de livres, magazines comptables nationaux et internationaux, résumés et sites internet. La conclusion principale démontre qu'il s'agit de normes dirigées à l'investisseur, qui priorisent $l^{\prime}$ aspect financier à l'économique, provenant du consensus entre différents courants théoriques développés pendant les dernières décennies, validés par la pratique des affaires et dont la finalité est de refléter l'essence économique des opérations et présenter une image fidèle de la situation financière $\mathrm{d}$ 'une entreprise. Les normes internationales d'information financière sont émises para l' International Accounting Standards Board (IASB) (avant International Accounting Standards Commite).

\section{Mots clés}

Normes, concepts comptables, principes comptables.

\section{Introducción}

Distintos eventos históricos relacionados con la realidad de las empresas originaron cambios en el uso de los sistemas contables en el mundo entre los siglos XVIII al XX, a saber:

1. la Revolución Industrial y su influencia en la contabilidad de costos y la contabilización de la depreciación;

2. el crecimiento y el desarrollo de los ferrocarriles;

3. la regulación gubernativa de los negocios; 
4. la imposición fiscal de las empresas;

5. el desarrollo de los gigantes industriales y financieros mediante fusiones, $y$

6. la influencia de la teoría económica.

Pero, ¿qué milagro llevó a la unificación de criterios contables entre el Instituto Americano de Contadores y la Bolsa de Valores de Nueva York?

La llamada Gran Depresión se originó en los Estados Unidos a partir de la caída de la Bolsa del 29 de octubre de 1929, día conocido como Martes Negro, aunque cinco días antes, el 24 de octubre, ya se había producido el Jueves Negro. Dicho desplome se extendió rápidamente a casi todos los países del mundo (Rothbard, 2013) debido al sobreprecio de los valores bursátiles, situación que provocó la aparición del movimiento de innovación contable. Esta corriente cuestionó por primera vez la total libertad de acción que existía en el campo contable, gran responsable por la falta de comparación de la información patrimonial que tenía que ser divulgada por las empresas de la época.

Esta dolorosa experiencia coadyuvó a la aparición en el mundo de los modelos de estados financieros que se obliga a publicar trimestralmente a las sociedades mercantiles, $y$ a exigir la presencia del Estado en el control de los registros públicos, la emisión de leyes sobre banca y seguros, códigos de comercio etc.

Tras cada debacle financiera, la demanda de cambios en la contabilidad ha sido una constante en la historia del mundo occidental, incluidos los gritos, las protestas y las demandas de soluciones urgentes y rápidas. Esta vez tampoco ha sido diferente. Aun cuando nadie bien informado ha culpado nunca a la contabilidad de los desastres económicos, especialmente los bursátiles, los esfuerzos renovadores tras cada crisis económica han influido en el ritmo del cambio contable. El reto esta vez será encontrar unos criterios contables que aborden la complejidad con que hoy en día se desarrolla el mundo de los negocios, que además han de ser válidos y aceptados universalmente (Pérez, 2010). 
¿Qué otros acontecimientos obligaron al cambio de los "criterios tradicionales" a los que ahora la International Accounting Standard Board (IASB) ${ }^{4}$ aplica en el mundo? Andersen Consulting (2000) nos informa sobre la presencia de los mercados de valores tecnológicos, que surgen ante la necesidad de dotar de financiación a empresas de tecnología de punta y con escasa historia de operaciones, organizaciones que generalmente no cumplen con los requisitos necesarios para cotizar en mercados normales. Estos mercados tienen condiciones de admisión diferentes de aquellos de los mercados tradicionales, por ejemplo, el requisito de obtención de beneficios se sustituye por un requisito de fondos propios, ventas o activos, y se exigen free floats mínimos.

El mercado Nasdaq nació de una iniciativa del Congreso de los Estados Unidos para regular el mercado de acciones over the counter (OTC o mercado no organizado). Creado en 1971 como el primer mercado electrónico del mundo, actualmente es el de mayor crecimiento de Estados Unidos y el segundo mayor en el mundo por capitalización bursátil después del NYSE. En 1963, la SEC (Comisión de Valores de Nueva York) llegó a la conclusión de que el mercado OTC era oscuro, fragmentado y que no contribuía a una creación eficiente de precios. La SEC dio el mandato de creación de un mercado automatizado a los intermediarios (National Association of Securities Dealers, NASD) $)^{5}$, y su construcción se inició en 1968. En 1971 empezó a funcionar bajo la nomenclatura de NASDAQ (Cotizaciones de la Asociación Nacional de Corredores de Valores) con más de 2500 valores. Este sería el primer mercado automatizado del mundo, frente a los mercados tradicionales de viva voz.

Pero el colmo de los problemas de información que las empresas transnacionales ya sentían se dio en 1993. Veamos la siguiente cita (Doupnik y Perera, 2007): "Daimler-Benz (ahora DaimlerChrysler) fue la primera compañía alemana que se inscribió en la Bolsa de Valores de Nueva York (NYSE), lo cual sucedió en 1993. Anteriormente las compañías alemanas

4 Se refiere a la Junta de Estándares Internacionales de Contabilidad.

5 Asociación Nacional de Corredores de Valores de Estados Unidos de Norteamérica. 
se habían negado a hacer los ajustes necesarios para reconciliar sus estados financieros, basados en leyes alemanas, con los principios de contabilidad generalmente aceptados de los Estados Unidos de Norteamérica. Después de algún compromiso con la SEC, y debido al fuerte deseo de Daimler por entrar al mercado de capitales estadounidenses (y por ser la primera compañía alemana en hacerlo), Daimler estuvo de acuerdo en cumplir con las regulaciones de la SEC. El hecho es que, en 1993, Daimler-Benz reportó una utilidad bajo los principios de contabilidad alemanes, pero una pérdida bajo los principios de contabilidad estadounidenses [hecho que] creó toda una conmoción en la comunidad financiera internacional, la cual fue de cerca de DM 2.5 miles de millones. En 1996, Daimler-Benz decidió abandonar los principios de contabilidad alemanes e implantar un sistema de contabilidad basado en los principios de contabilidad estadounidenses a nivel mundial" (Duopnik \& Perera, 2007).

El detonante de esta realidad fue que la profesión contable y los organismos mundiales a cargo del establecimiento de normas (llámese Organización de Naciones Unidas, la Unión Europea, la Asociación Internacional de Bolsas de Valores (IOSCO), la Federación Internacional de Contadores (IFAC)), dada la importancia de las actividades de la International Accounting Standards Board (IASB), la cual tomó el lugar del IASC en 2001, han estado bajo la presión de las compañías multinacionales, de las Bolsas de Valores, de los reguladores de valores, de instituciones internacionales de préstamos, como el Banco Mundial, para reducir la diversidad y armonizar los principios y las prácticas de contabilidad en el ámbito internacional.

El desarrollo de este trabajo de investigación, y que revisaremos a continuación, se enmarca en el ambiente descrito y contempla los siguientes puntos:

a. Los principios de contabilidad tradicionales.

b. Por qué resulta necesaria la contabilidad mundial.

c. La identificación de los postulados de contabilidad mundial. 


\section{Marco de referencia}

\section{Principios de contabilidad tradicionales}

Estos principios son los siguientes:

\section{Entidad}

Señala que la contabilidad está referida a las operaciones de una empresa, la cual es distinta a su dueño o empresarios, por lo que no corresponde mezclar ambos intereses. En este plano, la contabilidad pasa a constituir un principio ético, por lo que el contador deberá cautelar su cumplimiento evitando, por ejemplo, que los gastos particulares del propietario sean contabilizados como gastos de la empresa. Este principio pasa a ser un postulado básico, dado que las instituciones fiscales de todo el mundo la han reglamentado tan minuciosamente que ya nada queda al azar. Sin embargo, en las normas internacionales de contabilidad y/o normas internacionales de información financiera se le reconoce como "entidad económica".

\section{Empresa en marcha}

Principio mediante el cual se presume que no existe un límite de tiempo en la prolongación operacional de la entidad económica. Este principio se ha conservado como postulado básico en las normas internacionales de contabilidad con la denominación de "negocio en marcha".

\section{Hechos económicos}

Establece que la contabilidad registra solo hechos económicos, de modo que los estados contables se refieren a hechos, recursos y obligaciones económicas susceptibles de ser valorizados en términos monetarios. Este principio se ha mantenido como postulado básico en las normas internacionales de contabilidad como "sustancia económica".

\section{Moneda común denominador}

Obliga a la contabilidad a medir en términos monetarios, lo que permite reducir todos sus componentes heterogéneos a un común denominador. 
Este principio es recogido en la NIC n. ${ }^{\circ} 21$. Efecto de las variaciones en los tipos de cambio de la moneda extranjera (IAS 21, 2006). Como es de suponer, una entidad puede llevar a cabo actividades en el extranjero de dos maneras diferentes: puede realizar transacciones en moneda extranjera, o bien, puede tener negocios en el extranjero. Además, la entidad puede presentar sus estados financieros en una moneda extranjera, para lo cual define hasta cuatro tipos de moneda de acuerdo con las necesidades empresariales en el mundo global:

- Moneda de informe o presentación. Aquella elegida y utilizada por una entidad para presentar sus estados financieros.

- Moneda de registro. Es aquella en la cual la entidad mantiene sus registros contables, ya sea para fines legales o de información.

- Moneda extranjera. Es cualquier moneda distinta a la de registro, a la funcional o a la de informe de la entidad, según las circunstancias.

- Moneda funcional. Aquella con la que una entidad opera en su entorno económico primario, es decir, en el que principalmente genera y aplica efectivo.

Este principio pasa a ser un integrante del postulado de integridad.

\section{Periodo contable}

Considera que la vida de las empresas está dividida en periodos iguales y sucesivos, lo que facilita su control y la evaluación de sus actividades. Para el caso de Perú, el periodo contable de un año es el ejercicio fiscal que se inicia el $1 .{ }^{\circ}$ de enero y termina el 31 de diciembre. Este principio continúa como postulado básico en las normas internacionales de contabilidad, con el nombre de "asociación de costos y gastos con ingresos".

\section{Revelación suficiente o exposición}

Norma que, al término de cada periodo denominado ejercicio, deberá hacer una exposición acerca de la situación empresarial mediante la confección de estados contables. 
De acuerdo con la NIC n. ${ }^{\circ}$, dice (IAS 1, 2007, p. 537-577):

Establecer el marco global para la presentación de estados financieros para uso general, incluyendo lineamientos sobre su estructura y el contenido mínimo.

- Principios fundamentales que subyacen a la preparación de estados financieros, incluyendo la hipótesis de empresa en marcha, la uniformidad de la presentación y clasificación, el principio contable del devengamiento y la importancia relativa.

- Los activos y pasivos, así como los ingresos y gastos no pueden compensarse, salvo que la compensación esté permitida o sea exigida por otra NIIF.

- Debe presentarse información comparativa correspondiente al ejercicio anterior para los importes incluidos en los estados financieros y en la información complementaria.

- Generalmente, los estados financieros deben prepararse con una periodicidad anual. Si cambia la fecha de cierre del ejercicio y los estados financieros se presentan para un periodo distinto al año, será necesario informar de este hecho.

Un conjunto completo de estados financieros debe incluir:

- un estado de situación financiera,

- un estado del resultado integral,

- un estado de cambios en el patrimonio neto,

- un estado de flujos de efectivo y

- notas explicativas.

Principio que cambia a un requisito de calidad.

\section{Devengado}

Considera que la determinación de los resultados de operación y la posición financiera deben tomar en cuenta todos los recursos y las obligaciones del periodo, aunque estos hayan sido o no percibidos o pagados, es decir, debe considerar lo pagado y lo comprometido, con el objeto de que tanto 
los costos como los gastos puedan ser debidamente relacionados con los respectivos ingresos que generan.

Este principio permanece como postulado básico en las normas internacionales de información financiera en el marco de la contabilidad mundial, y en adelante se denominará "devengación contable".

\section{Realización}

Consiste en determinar los resultados económicos cuando la operación que los origina queda perfeccionada, estableciendo, en consecuencia, cuándo se debe considerar percibido un ingreso.

Este principio se sustituye en las normas internacionales de contabilidad por el postulado básico denominado "devengación contable", y se incorpora con un significado más amplio, aun cuando conserva su esencia, con el fin de fijar el momento de reconocimiento de los efectos derivados de las operaciones que lleva a cabo una entidad y de otros eventos que la afectan. Dicho cambio se instaura para adecuar la terminología conceptual al entorno internacional, dado que los organismos líderes emplean el término de "devengación contable" o "devengo contable" para establecer que el sistema de información contable opera sobre una base de acumulación más que sobre una base de cobro o pago de efectivo (Comisión de Principios Contables del Instituto Mexicano de Contadores, 2006).

\section{Prudencia}

Este principio general se puede expresar también de la siguiente manera: contabilizar todas las pérdidas cuando se conocen, y las ganancias solamente cuando se hayan realizado. También aclara que tiene lugar cuando el contador tiene dos o más opciones, pero este elegirá la que le muestre la cifra menor de dos valores de un activo relativo a una partida determinada. Esto significa que cuando se deba elegir entre dos valores para un elemento del activo, normalmente se debe optar por el más bajo, o bien, que una operación debe contabilizarse de tal modo que la alícuota del propietario sea menor. 
Tanto el Consejo de la Financial Accounting Standard Board ${ }^{6}$ (FASB, 2010) como el de General Agreed Accounting Principles of the United States of America (US- GAAP) (Van Hulle, 1992) prefirieron no incluir el conservadurismo (la prudencia) dentro del marco conceptual de las normas internacionales porque este no resultaba útil a los inversores, ya que podía ser manipulado para dar una información ficticia acerca de los estados financieros de las empresas y entrar así en conflicto con principios como la fidelidad de representación, la comparabilidad, la coherencia y la neutralidad; lo anterior ocasiona que los preparadores de la información la filtren, quitando así el derecho a los usuarios de decidir qué información es relevante o no (Silva y Azua, 2006). Principio que cambia al postulado de integridad.

\section{Costo como base de valuación}

Establece que el registro de las operaciones se basa en el valor económico que tienen los bienes y servicios, es decir, el "costo", ya sea de producción, adquisición o canje, el cual actúa como base para la valuación de estos.

El principio de valor histórico original se sustituye por el postulado de valuación, o valor razonable, el cual establece la forma de cuantificar las operaciones que una entidad lleva a cabo y otros eventos que la afectan. El postulado de valuación no busca profundizar sobre las posibles bases de valuación de los elementos de los estados financieros (Comisión de Principios de Contabilidad del Instituto Mexicano de Contadores, 2006).

\section{Consistencia o uniformidad}

Señala que los procedimientos de cuantificación utilizados deben ser uniformemente aplicados de un ejercicio a otro, de modo que cuando existan razones fundadas para cambiar de procedimientos, deberán informarse tanto el hecho como su efecto. Esto tiene una gran importancia, puesto que

6 Se refiere a la Junta de Estándares de Contabilidad Financiera de Estados Unidos de Norteamérica. 
uniforma los procedimientos y métodos aplicados, de modo que, si una operación específica es registrada de una forma determinada, cuando una operación similar vuelva a acontecer en el futuro, esta deberá ser registrada tal como la anterior.

Este principio se ha mantenido como postulado básico en las normas internacionales de información financiera en el marco de la contabilidad mundial, y en adelante se denominará "consistencia".

\section{Materialidad o importancia relativa}

Establece que para imputar las partidas, deberá tenerse en cuenta su importancia monetaria y/o vida útil significativa, ya que si, por ejemplo, se adquieren útiles de aseo, debido a que la importancia económica de estos elementos es relativamente baja a la vez que su vida útil es escasa, no tendría objeto imputarlos como bienes de la empresa, por cuanto serán rápidamente consumidos y pasarán a ser un gasto, por lo tanto, en lugar de ser activados como bienes, serán imputados directamente como gastos. La NIC n. ${ }^{\circ}$ 1. Presentación de Estados Financieros (2007, p. 546) lo redefine de esta manera: "Materialidad (o importancia relativa). La evaluación acerca de si una omisión o inexactitud puede influir en las decisiones económicas de los usuarios, considerándose así material o con importancia relativa, requiere tener en cuenta las características de tales usuarios".

El Marco conceptual para la preparación y presentación de la información financiera (IASB, 2010), en el párrafo 25 establece: "se supone que los usuarios tienen un conocimiento razonable de las actividades económicas y del mundo de los negocios, así como de su contabilidad, y también la voluntad de estudiar la información con razonable diligencia. En consecuencia, la evaluación necesita tener en cuenta, como puede esperarse, que, en términos razonables, usuarios con las características descritas se vean influidos al tomar decisiones económicas".

Principio que cambia a un requisito de calidad. 


\section{Partida doble}

Se constituye de:

a. los recursos de los que dispone la entidad para la realización de sus fines y

b. las fuentes de dichos recursos, que a su vez son la especificación de los derechos que sobre tales recursos existen, considerados en su conjunto.

Este principio se conserva como postulado básico en las normas internacionales de información financiera en el marco de la contabilidad mundial, como "dualidad económica".

\section{Equidad}

Principio fundamental que debe orientar la acción del profesional contable en todo momento. Se enuncia así: la equidad entre intereses opuestos debe ser una preocupación constante en contabilidad, puesto que los que sirven de o utilizan los datos contables pueden encontrarse ante el hecho de que los intereses particulares se hallen en conflicto.

En las normas internacionales de contabilidad se denomina también “integridad" y se enuncia de la siguiente manera (IASC, 1984):

Para ser confiable, la información contable contenida en los estados financieros debe ser neutral, es decir, libre de sesgos. Los estados financieros no serán neutrales si vía selección o presentación de la información se influencia la toma de decisiones o el juicio, a fin de lograr un resultado predeterminado.

\section{Postulados de contabilidad mundial}

Las NIC-NIIF ${ }^{7}$ constituyen un modelo que toma como punto de partida el principio contable de la empresa en funcionamiento, de manera que

7 Se refiere a normas internacionales de contabilidad y normas internacionales de información financiera. 
todas las normas que contiene van encaminadas a recoger el acontecer del día a día de los sujetos contables con un fin fundamental: la elaboración y presentación de los estados financieros del ejercicio económico.

El Consejo Mexicano para la Investigación y Desarrollo de Normas de Información Financiera (2006) explica que utilizan el término "normas de información financiera" en sustitución de los "principios de contabilidad generalmente aceptados" (PCGA) porque buscan enfatizar en el carácter normativo y obligatorio de dichas normas; el vocablo "principio" sugiere grados de rigidez, permanencia y universalidad, que no denotan la naturaleza dinámica de una NIF, en cambio, estas normas se van adaptando a los cambios experimentados en el entorno bajo el cual operan las entidades. Esto significa que unas NIF se van modificando, otras se eliminan, o bien, se van emitiendo nuevas disposiciones; por lo tanto, no debe considerarse lo afirmado en ellas como fundamento inamovible, signo de los tiempos que vivimos en una economía mundial inmersa en la volatilidad.

Tabla 1. Principios que pasan a ser postulados básicos en las normas internacionales de información financiera

\begin{tabular}{l|l}
\multicolumn{1}{c|}{ Principios contables } & \multicolumn{1}{c}{ Postulados básicos } \\
\hline Entidad & Entidad económica \\
\hline Empresa en marcha & Negocio en marcha \\
\hline Hechos económicos & Sustancia económica \\
\hline Costo como base de valuación & Valuación \\
\hline Devengado & \multirow{2}{*}{ Requisitos de calidad } \\
\cline { 1 - 1 } Realización & \\
\hline Revelación suficiente o exposición & Integridad \\
\hline Equidadialidad o importancia relativa & \\
\cline { 1 - 1 } Moneda común denominador & Asociación de costos y gastos con ingresos \\
\hline Prudencia &
\end{tabular}




\begin{tabular}{l|l}
\multicolumn{1}{c|}{ Principios contables } & \multicolumn{1}{c}{ Postulados básicos } \\
\hline Consistencia o uniformidad & Consistencia \\
\hline Partida doble & Dualidad económica \\
\hline
\end{tabular}

Fuente: elaboración propia con datos de la Comisión de Principios de Contabilidad. Instituto Mexicano de Contadores, 2006.

Sin embargo, el tema polémico del nuevo modelo es la aplicación del valor razonable (Gómez, 2009, p. 36):

Así, el desafío actual de la contabilidad es, por una parte, mantener su registro cronológico de las causas y efectos de los hechos económicos y, por la otra, reconocer las causas y efectos de la incertidumbre y del tiempo sobre los recursos y obligaciones de la entidad, actualizando los valores históricos o descontando los valores futuros para traerlos a valor presente, de manera de presentar sus estados financieros a valor razonable.

\section{Por qué resulta necesaria la contabilidad mundial}

Por muchas razones obvias, cuando se observa en perspectiva el mercado global.

- Para incrementar las ventas y las utilidades internacionales mediante el despliegue de ventajas comparativas y/o competitivas.

- Facilita el ingreso a mercados crecientes y/o rápidamente emergentes.

- Contribuye a la reducción de costos vía inversión extranjera directa y/o creación de subsidiarias aliadas con el capital nativo.

- Para proteger los mercados nacionales de los competidores extranjeros.

- Para proteger los mercados internacionales de los competidores locales.

- Para incrementar la adquisición de tecnología y de conocimientos administrativos globales.

- Facilita el comercio internacional de las importaciones y exportaciones.

- Proyecta a largo plazo el crecimiento de la empresa nacional en corporaciones multinacionales que, según definición de las Naciones Unidas, 
son empresas que poseen o controlan las instalaciones de producción o de servicios fuera del país donde están basadas (Organización de las Naciones Unidas, ONU, 1973).

- Ayuda a la inscripción en forma cruzada de sus acciones en Bolsas de Valores extranjeras.

- Para reducir los costos de financiamiento de futuros proyectos de inversión por ampliación de planta en cualquier parte del mundo. (Rugman y Hodggets, 1995).

\section{Objetivo de la investigación}

Examinar los nuevos postulados básicos a la luz de los principios de la contabilidad generalmente aceptados tradicionales.

\section{Metodología aplicada}

- Analítico-explicativa, porque consiste en descomponer los principios de contabilidad generalmente aceptados, separando cada una de las partes del todo para estudiarlas en forma individual con los nuevos postulados básicos que plantean las normas internacionales de información financiera. Se orienta enprofundizar en casos específicos y no en generalizar.

- De análisis documental, pues nos permitió obtener información principalmente de la revisión bibliográfica de libros, revistas de investigación contable internacionales, compendios y páginas web.

- De síntesis, para construir la relación causa-efecto entre los objetos que componen los elementos de la investigación.

\section{Enfoque}

Llegados a este nivel de análisis, queda clara la presencia de dos modelos dominantes de contabilidad que se usan en cuanto al manejo de la información internacional en el mundo desarrollado: 
1. El modelo europeo-continental. El principal objetivo de la contabilidad europea ha sido proteger a los acreedores, pues la tradicional forma de financiación de las empresas europeas ha sido la financiación ajena, sobre todo vinculada a entidades de crédito, que promueve un nivel débil de financiamiento externo de capital.

2. El modelo británico-estadounidense. El sistema contable de EE. UU. deriva del modelo anglosajón, en el cual, el componente financiero pesa más que el legal. Una consecuencia directa de ello es que nuestro sistema contable ha estado más ligado a normas fiscales, mientras que el norteamericano no. La normativa US $\mathrm{GAAP}^{8}$ es muy voluminosa y está basada en normas e interpretaciones muy detalladas y pormenorizadas, en las que hay un nivel fuerte de financiamiento externo de capital. Sin embargo, el principal objetivo de la contabilidad americana es ofrecer información a los inversores, pues su tradicional fuente de financiación son los mercados de valores. Está claramente orientado al inversor, de acuerdo con la idea de que si la empresa ha perdido fondos en el mercado, debe informar adecuadamente al que se los prestó, para que este pueda decidir sus inversiones con base en el riesgo. De ahí el enfoque de balance, la primacía de los flujos de caja y las importantes exigencias de información a los mercados. No se piensa de forma prioritaria en la Hacienda Pública ni en los acreedores, por ello, no se impone necesariamente el método del coste histórico, sino el fair value (valor razonable); tampoco se generan plusvalías ocultas derivadas de la aplicación estricta del criterio de prudencia. En este modelo no tienen cabida, por tanto, nuestras tradicionales provisiones "por si acaso".

Y si aún quedan dudas de la orientación final que tienen las normas internacionales, estas priorizan la liquidez a la rentabilidad. Veamos las ventajas que tiene la promoción del mercado de capitales:

1. Provee a las compañías la facilidad para conseguir el capital necesario para iniciar una inversión o negocio.

8 Principios de contabilidad generalmente aceptados de Estados Unidos de Norteamérica. 
2. Canaliza los ahorros de las economías domésticas hacia las necesidades de inversión de los distintos sectores económicos, dando como resultado un fuerte desarrollo económico y altos niveles de productividad.

3. Facilita el crecimiento de las empresas por medio de fusiones, nuevas inversiones, expansión del mercado, operaciones que necesitan de grandes sumas de capital proveniente de la institución bursátil.

4. Al ser las acciones un título de propiedad sobre una parte del capital de una empresa, la obliga a mejorar sus estándares de eficacia de la gerencia para satisfacer las demandas de estos accionistas, además de obligar a los gobiernos corporativos de las empresas a proporcionar información económico-financiera de la forma más transparente posible.

5. Crea oportunidades de inversión para los pequeños inversores.

6. Actúa como el barómetro de la economía, ya que la estabilidad económica se refleja en los precios de las acciones, los cuales actuarán manteniéndose o aumentando en el caso de bonanzas económicas. Sin embargo, en épocas de crisis financieras o recesión, se produce un desplome general de todos los índices bursátiles.

\section{Resultados y discusión}

Los escándalos financieros acaecidos en los Estados Unidos a finales de 2001 levantaron una serie de críticas que demandaban a reguladores y emisores de normas contables la revisión y mejora de tales normas.

En 2002, el Congreso de los Estados Unidos emitió la Ley SarbanesOxley, orientada a restaurar la confianza del inversor. La ley no tenía como único objetivo la identificación de los "malos actores" y de sus "malas prácticas", sino que llegó a cuestionar si las normas contables no eran las responsables de tales conductas. El Congreso de EE. UU. ordenó a la SEC llevar a cabo una revisión de los enfoques existentes para emitir normas de contabilidad, a fin de determinar el camino más adecuado para cumplir con lo establecido por la citada ley (reglas o principios generales). 
Según Ramírez (2004, p. 6):

En el año 2001, el IASC se convirtió en la Fundación IASCF, y la labor de expedir estándares internacionales de información financiera pasó a la IASB (International Accounting Standards Board). A partir del año 2001, la IASB inició el proceso para emitir las IFRS o NIIF, que sustituyen a las IAS o NIC, cambiando el enfoque de norma de contabilidad por estándar de información financiera.

Las IFRS o NIIF, que sustituyen a las IAS o NIC, no son solo un cambio de nombre, sino que implican el paso de un esquema de preparación y presentación de $\mathrm{E} / \mathrm{F}$ basado en el registro a otro completamente diferente de medición y presentación de reportes sobre el desempeño financiero.

Es el fin del conservadurismo y del costo como base de valuación, para ingresar a los postulados básicos, cuya característica fundamental es la adaptación a los cambios experimentados en el entorno, como la volatilidad de la economía mundial de la mano del fair value o "valor razonable" (el precio que sería recibido por vender un activo o pagado por transferir un pasivo en una transacción ordenada entre participantes del mercado en la fecha de la medición, es decir, un precio de salida). Esa definición de valor razonable enfatiza en que es una medición basada en el mercado, no una medida específica de una entidad (IFRS 13, NIIF 13, 2012).

- ¿Contribuirá el valor razonable a mejorar el patrimonio de las empresas en el mundo?

- ¿Hemos claudicado los profesionales contables ante la eliminación del costo histórico?

- ¿Es mejor el enfoque probabilístico que el de prudencia para evaluar los activos y pasivos contingentes?

- ¿No nos obliga la dualidad contable a priorizar lo económico-financiero sobre solamente liquidez? 


\section{Conclusiones}

Se demostró que la diversidad contable produce diferencias significativas en la valuación de los ingresos y el patrimonio en la presentación de estados financieros entre países, como lo demuestra el caso de la Daimler-Benz, que reportó utilidad aplicando los principios de contabilidad alemanes, pero una pérdida con los estadounidenses. Se evidencia que son normas orientadas al inversor, que priorizan lo financiero sobre lo económico, provenientes del consenso alcanzado por diferentes corrientes teóricas desarrolladas en las últimas décadas, validadas por la práctica de los negocios y cuya finalidad es reflejar la esencia económica de las operaciones y presentar una imagen fiel de la situación financiera de la empresa.

$\mathrm{Al}$ analizar los dos modelos dominantes de contabilidad (el europeocontinental y el británico-estadounidense), encontramos que el primero protegía el interés de los acreedores, mientras que, en el segundo, el componente financiero pesa más que el legal, y en él se financian las inversiones en el mercado de valores, por lo tanto, es más importante ofrecer información a los inversionistas para generar trabajo, mejorar el nivel de vida de las personas y promover ahorro e inversión.

En el contexto académico, en reemplazo de los principios del siglo pasado promovamos los "postulados básicos", que son los siguientes: entidad económica, negocio en marcha, sustancia económica, devengación contable, integridad, asociación de costos y gastos con ingresos, valuación, consistencia y dualidad económica.

\section{Referencias bibliográficas}

Andersen Consulting. (2000). Mercados de valores tecnológicos. Recuperado de ciberconta.unizar.es/ftp/pub/docs/bsch/pdf/C.pdf

Doupnik, T., \& Perera, H. (2007). Contabilidad internacional. México: Editorial McGraw-Hill/Interamericana Editores. 
Gómez, A. (2009). Métodos de valuación a valor razonables y su auditoría. Santiago. Chile: Universidad de las Américas.

Comisión de Principios de Contabilidad del Instituto Mexicano de Contadores. (2006). NIF-A1. Estructura de las normas de información financiera. México: Autor.

Comisión de Principios de Contabilidad del Instituto Mexicano de Contadores. (2006). NIF A-2. Postulados básicos. México: Autor.

IASB. (2007). IAS1 NIC n..$^{\circ}$. Presentación de estados financieros. Londres: Autor. IASB. (2010). Marco conceptual para la preparación y presentación de la información financiera. Londres: Autor.

IASCF. (2006). IAS 21 NIC n. ${ }^{\circ} 21$. Efecto de las variaciones en los tipos de cambio de la moneda extranjera. Recuperado de http://www.normasinternacionalesdecontabilidad.es/nic/pdf/NIC21.pdf

IASC. (1984). IAS 1 NIC $n .^{\circ} 1$. Presentación de estados financieros. Londres: Autor. IFRS - NIIF. (2012). NIIF n. ${ }^{\circ}$ 13. Medición del valor razonable. Recuperado de http:// www.ifrs.org/IFRSs/Documents/IFRS13sp.pdf

Organización de las Naciones Unidas. (1973). Multinational Corporations in World Development. Nueva York: Autor.

Pérez, J. (2010). Normas internacionales de información financiera NIC / NIIF. Una verdadera revolución: Efectos contables de la convergencia financiera mundial. CISS Técnica Contable, 62(731), 44-52. Recuperado de http://www.elcriterio. com/seccion_articulos/tecnicacontable_jorge_perez3.pdf

Ramírez E. (2004). Bases y fundamentos de la contabilidad financiera internacional. Recuperado de http://www.conalcon.com/pdf/art.\%202\%20conalcon.pdf

Rothbard, M. (2013). La Gran Depresión. Recuperado de http://es.wikipedia.org/ wiki/Crisis_de_1929

Rugman, A. M., \& Hodggets, R. M. (1995). International Business: A Strategic Management Approach. Nueva York: McGraw-Hill.

Silva, B. y Azua, D. (2006). Alcances sobre el concepto de valor razonable. Valparaíso, Chile: Escuela de Comercio. Pontificia Universidad Católica.

Van Hulle, K. (1992). Harmonization of accounting standards: A view from the European Community. European Accounting Review 1(1), 161-172. 